Research Article

\title{
Affine Ricci Solitons of Three-Dimensional Lorentzian Lie Groups
}

\author{
Yong Wang* \\ School of Mathematics and Statistics, Northeast Normal University, Changchun 130024, China
}

ARTICLE INF O
Article History
Received 28 December 2020
Accepted 28 January 2021
Keywords
Canonical connections
Kobayashi-Nomizu connections
perturbed canonical connections
perturbed Kobayashi-Nomizu
connections
affine Ricci solitons
three-dimensional Lorentzian
Lie groups
2010 Mathematics Subject
Classification
$53 C 40$
53 C42

\section{ARTICLE INFO}

Received 28 December 2020

Keywords

connections

perturbed canonic

three-dimensional Lorentzian

2010 Mathematics Subject

$53 \mathrm{C} 42$

\section{ABSTRACT}

In this paper, we classify affine Ricci solitons associated to canonical connections and Kobayashi-Nomizu connections and perturbed canonical connections and perturbed Kobayashi-Nomizu connections on three-dimensional Lorentzian Lie groups with some product structure.

(C) 2021 The Author. Published by Atlantis Press B.V. This is an open access article distributed under the CC BY-NC 4.0 license (http://creativecommons.org/licenses/by-nc/4.0/).

\section{INTRODUCTION}

The concept of the Ricci soliton is introduced by Hamilton in [9], which is a natural generalization of Einstein metrics. Study of Ricci soliton over different geometric spaces is one of interesting topics in geometry and mathematical physics. In particular, it has become more important after G. Perelman applied Ricci solitons to solve the long standing Poincare conjecture. In [10,13,15-18], Einstein manifolds associated to affine connections (especially semi-symmetric metric connections and semi-symmetric non-metric connections) were studied (see the definition 3.2 in [18] and the definition 3.1 in [10]). It is natural to study Ricci solitons associated to affine connections. Affine Ricci solitons had been introduced and studied, for example, see $[6,8,11,12,14]$.

Our motivation is to find more examples of affine Ricci solitons. A three-dimensional Lie group $G_{i}(i=1, \cdots, 7)$ is a sub-Riemannian manifold. In [1], Balogh, Tyson and Vecchi applied a Riemannian approximation scheme to get a Gauss-Bonnet theorem in the Heisenberg group $\mathbb{H}^{3}$. Let $T \mathbb{H}^{3}=\operatorname{span}\left\{e_{1}, e_{2}, e_{3}\right\}$, then they took the distribution $H=\operatorname{span}\left\{e_{1}, e_{2}\right\}$ and $H^{\perp}=\operatorname{span}\left\{e_{3}\right\}$ (for details, see [1]). Similarly in [20], for the affine group and the group of rigid motions of the Minkowski plane, we took the similar distributions. In [21], for the Lorentzian Heisenberg group, we also took the similar construction. Motivated by [1,20,21], we consider the similar distribution $H=\operatorname{span}\left\{e_{1}, e_{2}\right\}$ and $H^{\perp}=\operatorname{span}\left\{e_{3}\right\}$ for the three dimensional Lorentzian Lie group $G_{i}(i=1, \cdots, 7)$. Then for the above distribution, we have a natural product structure $J: J e_{1}=e_{1}, J e_{2}=e_{2}, J e_{3}=-e_{3}$. In [7], Etayo and Santamaria studied some affine connections on manifolds with the product structure or the complex structure. In particular, the canonical connection and the Kobayashi-Nomizu connection for a product structure were studied. So we consider the canonical connection and the Kobayashi-Nomizu connection associated to the above distribution on the $G_{i}$ and get affine Ricci solitons associated to the canonical connection and the Kobayashi-Nomizu connection. In particular, from our results, we can get affine Einstein manifolds associated to the canonical connection and the Kobayashi-Nomizu connection. It is interesting to consider relations between affine Ricci solitons associated to the canonical connection and the KobayashiNomizu connection and Ricci solitons associated to the Levi-Civita connection. It is also interesting to study affine Ricci solitons associated to other affine connections, for example, Schouten-Van Kampen connections and Vranceanu connections associated to the above product structure and semi-symmetric connections.

*Email: wangy581@nenu.edu.cn

Data availability statement: The authors confirm that the data supporting the findings of this study are available within the article. 
By the canonical connection and the Kobayashi-Nomizu connection on three-dimensional Lorentzian Lie groups, we obtain some examples of affine Ricci solitons. But we find that the coefficient $\lambda$ of the metric tensor $g$ in the Ricci soliton equation (see (3.13) and (3.14)) is always zero for these obtained examples. In order to obtain more interesting examples with the non zero coefficient $\lambda$, we introduce perturbed canonical connections and perturbed Kobayashi-Nomizu connections in Section 4. Using these perturbed connections, we get some examples of affine Ricci solitons with the non zero coefficient $\lambda$.

In [3], Calvaruso studied three-dimensional generalized Ricci solitons, both in Riemannian and Lorentzian settings. He determined their homogeneous models, classifying left-invariant generalized Ricci solitons on three-dimensional Lie groups. Then it is natural to classify affine Ricci solitons on three-dimensional Lie groups. In [19], we introduced a particular product structure on three-dimensional Lorentzian Lie groups and we computed canonical connections and Kobayashi-Nomizu connections and their curvature on three-dimensional Lorentzian Lie groups with this product structure. We defined algebraic Ricci solitons associated to canonical connections and Kobayashi-Nomizu connections. We classified algebraic Ricci solitons associated to canonical connections and Kobayashi-Nomizu connections on threedimensional Lorentzian Lie groups with this product structure. In this paper, we classify affine Ricci solitons associated to canonical connections and Kobayashi-Nomizu connections and perturbed canonical connections and perturbed Kobayashi-Nomizu connections on three-dimensional Lorentzian Lie groups with this product structure.

In Section 2, we recall the classification of three-dimensional Lorentzian Lie groups. In Section 3, we classify affine Ricci solitons associated to canonical connections and Kobayashi-Nomizu connections on three-dimensional Lorentzian Lie groups with this product structure. In Section 4, we classify affine Ricci solitons associated to perturbed canonical connections and perturbed Kobayashi-Nomizu connections on three-dimensional Lorentzian Lie groups with this product structure.

\section{THREE-DIMENSIONAL LORENTZIAN LIE GROUPS}

In this section, we recall the classification of three-dimensional Lorentzian Lie groups in [4,5](also see Theorems 2.1 and 2.2 in [2]).

Theorem 2.1. Let $(G, g)$ be a three-dimensional connected unimodular Lie group, equipped with a left-invariant Lorentzian metric. Then there exists a pseudo-orthonormal basis $\left\{e_{1}, e_{2}, e_{3}\right\}$ with $e_{3}$ timelike such that the Lie algebra of $G$ is one of the following:

$\left(\mathfrak{g}_{1}\right)$ :

$\left(\mathfrak{g}_{2}\right)$ :

$$
\left[e_{1}, e_{2}\right]=\alpha e_{1}-\beta e_{3}, \quad\left[e_{1}, e_{3}\right]=-\alpha e_{1}-\beta e_{2}, \quad\left[e_{2}, e_{3}\right]=\beta e_{1}+\alpha e_{2}+\alpha e_{3}, \quad \alpha \neq 0
$$

$\left[e_{1}, e_{2}\right]=\gamma e_{2}-\beta e_{3}, \quad\left[e_{1}, e_{3}\right]=-\beta e_{2}-\gamma e_{3}, \quad\left[e_{2}, e_{3}\right]=\alpha e_{1}, \quad \gamma \neq 0$

$\left(\mathfrak{g}_{3}\right)$ :

$\left(\mathfrak{g}_{4}\right)$ :

$$
\left[e_{1}, e_{2}\right]=-\gamma e_{3}, \quad\left[e_{1}, e_{3}\right]=-\beta e_{2}, \quad\left[e_{2}, e_{3}\right]=\alpha e_{1}
$$

$$
\left[e_{1}, e_{2}\right]=-e_{2}+(2 \eta-\beta) e_{3}, \quad \eta=1 \text { or }-1, \quad\left[e_{1}, e_{3}\right]=-\beta e_{2}+e_{3}, \quad\left[e_{2}, e_{3}\right]=\alpha e_{1}
$$

Theorem 2.2. Let $(G, g)$ be a three-dimensional connected non-unimodular Lie group, equipped with a left-invariant Lorentzian metric. Then there exists a pseudo-orthonormal basis $\left\{e_{1}, e_{2}, e_{3}\right\}$ with $e_{3}$ timelike such that the Lie algebra of $G$ is one of the following:

$\left(\mathfrak{g}_{5}\right)$ :

$$
\left[e_{1}, e_{2}\right]=0, \quad\left[e_{1}, e_{3}\right]=\alpha e_{1}+\beta e_{2}, \quad\left[e_{2}, e_{3}\right]=\gamma e_{1}+\delta e_{2}, \quad \alpha+\delta \neq 0, \quad \alpha \gamma+\beta \delta=0
$$

$\left(\mathfrak{g}_{6}\right)$ :

$$
\left[e_{1}, e_{2}\right]=\alpha e_{2}+\beta e_{3}, \quad\left[e_{1}, e_{3}\right]=\gamma e_{2}+\delta e_{3}, \quad\left[e_{2}, e_{3}\right]=0, \quad \alpha+\delta \neq 0, \quad \alpha \gamma-\beta \delta=0
$$

$\left(\mathfrak{g}_{7}\right)$ :

$$
\left[e_{1}, e_{2}\right]=-\alpha e_{1}-\beta e_{2}-\beta e_{3}, \quad\left[e_{1}, e_{3}\right]=\alpha e_{1}+\beta e_{2}+\beta e_{3}, \quad\left[e_{2}, e_{3}\right]=\gamma e_{1}+\delta e_{2}+\delta e_{3}, \quad \alpha+\delta \neq 0, \quad \alpha \gamma=0
$$

\section{AFFINE RICCI SOLITONS ASSOCIATED TO CANONICAL CONNECTIONS AND KOBAYASHI-NOMIZU CONNECTIONS ON THREE-DIMENSIONAL LORENTZIAN LIE GROUPS}

Throughout this paper, we shall by $\left\{G_{i}\right\}_{i=1, \ldots, 7}$, denote the connected, simply connected three-dimensional Lie group equipped with a left-invariant Lorentzian metric $g$ and having Lie algebra $\{\mathfrak{g}\}_{i=1, \ldots, 7}$. Let $\nabla$ be the Levi-Civita connection of $G_{i}$ and $R$ its curvature tensor, 
taken with the convention

$$
R(X, Y) Z=\nabla_{X} \nabla_{Y} Z-\nabla_{Y} \nabla_{X} Z-\nabla_{[X, Y]} Z
$$

The Ricci tensor of $\left(G_{i}, g\right)$ is defined by

$$
\rho(X, Y)=-g\left(R\left(X, e_{1}\right) Y, e_{1}\right)-g\left(R\left(X, e_{2}\right) Y, e_{2}\right)+g\left(R\left(X, e_{3}\right) Y, e_{3}\right),
$$

where $\left\{e_{1}, e_{2}, e_{3}\right\}$ is a pseudo-orthonormal basis, with $e_{3}$ timelike. We define a product structure $J$ on $G_{i}$ by

$$
J e_{1}=e_{1}, J e_{2}=e_{2}, J e_{3}=-e_{3},
$$

then $J^{2}=$ id and $g\left(J e_{j}, J e_{j}\right)=g\left(e_{j}, e_{j}\right)$. By [7], we define the canonical connection and the Kobayashi-Nomizu connection as follows:

$$
\begin{aligned}
& \nabla_{X}^{0} Y=\nabla_{X} Y-\frac{1}{2}\left(\nabla_{X} J\right) J Y, \\
& \nabla_{X}^{1} Y=\nabla_{X}^{0} Y-\frac{1}{4}\left[\left(\nabla_{Y} J\right) J X-\left(\nabla_{J Y} J\right) X\right] .
\end{aligned}
$$

We define

$$
\begin{aligned}
& R^{0}(X, Y) Z=\nabla_{X}^{0} \nabla_{Y}^{0} Z-\nabla_{Y}^{0} \nabla_{X}^{0} Z-\nabla_{[X, Y]}^{0} Z, \\
& R^{1}(X, Y) Z=\nabla_{X}^{1} \nabla_{Y}^{1} Z-\nabla_{Y}^{1} \nabla_{X}^{1} Z-\nabla_{[X, Y]}^{1} Z .
\end{aligned}
$$

The Ricci tensors of $\left(G_{i}, g\right)$ associated to the canonical connection and the Kobayashi-Nomizu connection are defined by

$$
\begin{aligned}
& \rho^{0}(X, Y)=-g\left(R^{0}\left(X, e_{1}\right) Y, e_{1}\right)-g\left(R^{0}\left(X, e_{2}\right) Y, e_{2}\right)+g\left(R^{0}\left(X, e_{3}\right) Y, e_{3}\right), \\
& \rho^{1}(X, Y)=-g\left(R^{1}\left(X, e_{1}\right) Y, e_{1}\right)-g\left(R^{1}\left(X, e_{2}\right) Y, e_{2}\right)+g\left(R^{1}\left(X, e_{3}\right) Y, e_{3}\right) .
\end{aligned}
$$

Let

$$
\widetilde{\rho}^{0}(X, Y)=\frac{\rho^{0}(X, Y)+\rho^{0}(Y, X)}{2},
$$

and

$$
\widetilde{\rho}^{1}(X, Y)=\frac{\rho^{1}(X, Y)+\rho^{1}(Y, X)}{2} .
$$

Since $\left(L_{V} g\right)(Y, Z):=g\left(\nabla_{Y} V, Z\right)+g\left(Y, \nabla_{Z} V\right)$, we let

$$
\left(L_{V}^{j} g\right)(Y, Z):=g\left(\nabla_{Y}^{j} V, Z\right)+g\left(Y, \nabla_{Z}^{j} V\right)
$$

for $j=0,1$ and vector fields $V, Y, Z$.

Definition 3.1. $\left(G_{i}, g, J\right)$ is called the affine Ricci soliton associated to the connection $\nabla^{0}$ if it satisfies

$$
\left(L_{V}^{0} g\right)(Y, Z)+2 \widetilde{\rho}^{0}(Y, Z)+2 \lambda g(Y, Z)=0
$$

where $\lambda$ is a real number and $V=\lambda_{1} e_{1}+\lambda_{2} e_{2}+\lambda_{3} e_{3}$ and $\lambda_{1}, \lambda_{2}, \lambda_{3}$ are real numbers. $\left(G_{i}, g, J\right)$ is called the affine Ricci soliton associated to the connection $\nabla^{1}$ if it satisfies

$$
\left(L_{V}^{1} g\right)(Y, Z)+2 \widetilde{\rho}^{1}(Y, Z)+2 \lambda g(Y, Z)=0
$$

By (2.25) in [19], we have for $\left(G_{1}, g, J, \nabla^{0}\right)$

$$
\begin{array}{ll}
\widetilde{\rho}^{0}\left(e_{1}, e_{1}\right)=-\left(\alpha^{2}+\frac{\beta^{2}}{2}\right), & \widetilde{\rho}^{0}\left(e_{1}, e_{2}\right)=0, \\
\widetilde{\rho}^{0}\left(e_{1}, e_{3}\right)=\frac{\alpha \beta}{4}, & \widetilde{\rho}^{0}\left(e_{2}, e_{2}\right)=-\left(\alpha^{2}+\frac{\beta^{2}}{2}\right), \\
\widetilde{\rho}^{0}\left(e_{2}, e_{3}\right)=\frac{\alpha^{2}}{2}, & \widetilde{\rho}^{0}\left(e_{3}, e_{3}\right)=0 .
\end{array}
$$

By Lemma 2.4 in [19] and (3.12), we have for $\left(G_{1}, g, J, \nabla^{0}, V\right)$

$$
\left(L_{V}^{0} g\right)\left(e_{1}, e_{1}\right)=2 \lambda_{2} \alpha, \quad\left(L_{V}^{0} g\right)\left(e_{1}, e_{2}\right)=-\lambda_{1} \alpha
$$




$$
\begin{array}{ll}
\left(L_{V}^{0} g\right)\left(e_{1}, e_{3}\right)=-\frac{\beta}{2} \lambda_{2}, & \left(L_{V}^{0} g\right)\left(e_{2}, e_{2}\right)=0, \\
\left(L_{V}^{0} g\right)\left(e_{2}, e_{3}\right)=\frac{\beta}{2} \lambda_{1}, & \left(L_{V}^{0} g\right)\left(e_{3}, e_{3}\right)=0 .
\end{array}
$$

If $\left(G_{1}, g, J, V\right)$ is an affine Ricci soliton associated to the connection $\nabla^{0}$, then by (3.13), we have

$$
\left\{\begin{array}{l}
2 \lambda_{2} \alpha-2 \alpha^{2}-\beta^{2}+2 \lambda=0 \\
\lambda_{1} \alpha=0 \\
-\beta \lambda_{2}+\alpha \beta=0 \\
-2 \alpha^{2}-\beta^{2}+2 \lambda=0 \\
\frac{\beta}{2} \lambda_{1}+\alpha^{2}=0 \\
\lambda=0
\end{array}\right.
$$

Solve (3.17), we have

Theorem 3.2. $\left(G_{1}, g, J, V\right)$ is not an affine Ricci soliton associated to the connection $\nabla^{0}$.

By (2.33) in [19], we have for $\left(G_{1}, g, J, \nabla^{1}\right)$

$$
\begin{array}{ll}
\widetilde{\rho}^{1}\left(e_{1}, e_{1}\right)=-\left(\alpha^{2}+\beta^{2}\right), & \widetilde{\rho}^{1}\left(e_{1}, e_{2}\right)=\alpha \beta, \\
\widetilde{\rho}^{1}\left(e_{1}, e_{3}\right)=-\frac{\alpha \beta}{2}, & \widetilde{\rho}^{1}\left(e_{2}, e_{2}\right)=-\left(\alpha^{2}+\beta^{2}\right), \\
\widetilde{\rho}^{1}\left(e_{2}, e_{3}\right)=\frac{\alpha^{2}}{2}, & \widetilde{\rho}^{1}\left(e_{3}, e_{3}\right)=0 .
\end{array}
$$

By Lemma 2.8 in [19] and (3.12), we have for $\left(G_{1}, g, J, \nabla^{1}, V\right)$

$$
\begin{array}{lll}
\left(L_{V}^{1} g\right)\left(e_{1}, e_{1}\right)=2 \lambda_{2} \alpha, & \left(L_{V}^{1} g\right)\left(e_{1}, e_{2}\right)=-\lambda_{1} \alpha, \\
\left(L_{V}^{1} g\right)\left(e_{1}, e_{3}\right)=\lambda_{1} \alpha-\beta \lambda_{2}, & \left(L_{V}^{1} g\right)\left(e_{2}, e_{2}\right)=0, \\
\left(L_{V}^{1} g\right)\left(e_{2}, e_{3}\right)=\beta \lambda_{1}-\alpha \lambda_{2}-\alpha \lambda_{3}, & & \left(L_{V}^{1} g\right)\left(e_{3}, e_{3}\right)=0 .
\end{array}
$$

If $\left(G_{1}, g, J, V\right)$ is an affine Ricci soliton associated to the connection $\nabla^{1}$, then by (3.14), we have

$$
\left\{\begin{array}{l}
\lambda_{2} \alpha-\alpha^{2}-\beta^{2}+\lambda=0 \\
-\lambda_{1} \alpha+2 \alpha \beta=0 \\
\lambda_{1} \alpha-\beta \lambda_{2}-\alpha \beta=0 \\
-\alpha^{2}-\beta^{2}+\lambda=0 \\
\beta \lambda_{1}-\alpha \lambda_{2}-\alpha \lambda_{3}+\alpha^{2}=0 \\
\lambda=0
\end{array}\right.
$$

Solve (3.20), we have

Theorem 3.3. $\left(G_{1}, g, J, V\right)$ is not an affine Ricci soliton associated to the connection $\nabla^{1}$.

By (2.44) in [19], we have for $\left(G_{2}, g, J, \nabla^{0}\right)$

$$
\begin{array}{ll}
\widetilde{\rho}^{0}\left(e_{1}, e_{1}\right)=-\left(\gamma^{2}+\frac{\alpha \beta}{2}\right), & \widetilde{\rho}^{0}\left(e_{1}, e_{2}\right)=0, \\
\widetilde{\rho}^{0}\left(e_{1}, e_{3}\right)=0, & \widetilde{\rho}^{0}\left(e_{2}, e_{2}\right)=-\left(\gamma^{2}+\frac{\alpha \beta}{2}\right), \\
\widetilde{\rho}^{0}\left(e_{2}, e_{3}\right)=\frac{\beta \gamma}{2}-\frac{\alpha \gamma}{4}, & \widetilde{\rho}^{0}\left(e_{3}, e_{3}\right)=0 .
\end{array}
$$

By Lemma 2.14 in [19] and (3.12), we have for $\left(G_{2}, g, J, \nabla^{0}, V\right)$

$$
\begin{array}{ll}
\left(L_{V}^{0} g\right)\left(e_{1}, e_{1}\right)=0, & \left(L_{V}^{0} g\right)\left(e_{1}, e_{2}\right)=\lambda_{2} \gamma \\
\left(L_{V}^{0} g\right)\left(e_{1}, e_{3}\right)=-\frac{\alpha}{2} \lambda_{2}, & \left(L_{V}^{0} g\right)\left(e_{2}, e_{2}\right)=-2 \gamma \lambda_{1}, \\
\left(L_{V}^{0} g\right)\left(e_{2}, e_{3}\right)=\frac{\alpha}{2} \lambda_{1}, & \left(L_{V}^{0} g\right)\left(e_{3}, e_{3}\right)=0 .
\end{array}
$$


If $\left(G_{2}, g, J, V\right)$ is an affine Ricci soliton associated to the connection $\nabla^{0}$, then by (3.13), we have

$$
\left\{\begin{array}{l}
-\left(\gamma^{2}+\frac{\alpha \beta}{2}\right)+\lambda=0, \\
\lambda_{2} \gamma=0 \\
\alpha \lambda_{2}=0 \\
-\gamma \lambda_{1}-\left(\gamma^{2}+\frac{\alpha \beta}{2}\right)+\lambda=0 \\
\frac{\alpha}{2} \lambda_{1}+2\left(\frac{\beta \gamma}{2}-\frac{\alpha \gamma}{4}\right)=0 \\
\lambda=0
\end{array}\right.
$$

Solve (3.23), we have

Theorem 3.4. $\left(G_{2}, g, J, V\right)$ is not an affine Ricci soliton associated to the connection $\nabla^{0}$.

By (2.54) in [19], we have for $\left(G_{2}, g, J, \nabla^{1}\right)$

$$
\begin{array}{ll}
\tilde{\rho}^{1}\left(e_{1}, e_{1}\right)=-\left(\beta^{2}+\gamma^{2}\right), & \widetilde{\rho}^{1}\left(e_{1}, e_{2}\right)=0, \\
\widetilde{\rho}^{1}\left(e_{1}, e_{3}\right)=0, & \widetilde{\rho}^{1}\left(e_{2}, e_{2}\right)=-\left(\gamma^{2}+\alpha \beta\right), \\
\widetilde{\rho}^{1}\left(e_{2}, e_{3}\right)=-\frac{\alpha \gamma}{2}, & \widetilde{\rho}^{1}\left(e_{3}, e_{3}\right)=0 .
\end{array}
$$

By Lemma 2.18 in [19] and (3.12), we have for $\left(G_{2}, g, J, \nabla^{1}, V\right)$

$$
\begin{array}{ll}
\left(L_{V}^{1} g\right)\left(e_{1}, e_{1}\right)=0, & \left(L_{V}^{1} g\right)\left(e_{1}, e_{2}\right)=\lambda_{2} \gamma, \\
\left(L_{V}^{1} g\right)\left(e_{1}, e_{3}\right)=-\alpha \lambda_{2}+\gamma \lambda_{3}, & \left(L_{V}^{1} g\right)\left(e_{2}, e_{2}\right)=-2 \gamma \lambda_{1}, \\
\left(L_{V}^{1} g\right)\left(e_{2}, e_{3}\right)=\lambda_{1} \beta, & \left(L_{V}^{1} g\right)\left(e_{3}, e_{3}\right)=0 .
\end{array}
$$

If $\left(G_{2}, g, J, V\right)$ is an affine Ricci soliton associated to the connection $\nabla^{1}$, then by (3.14), we have

$$
\left\{\begin{array}{l}
-\beta^{2}-\gamma^{2}+\lambda=0 \\
\lambda_{2} \gamma=0 \\
-\alpha \lambda_{2}+\gamma \lambda_{3}=0 \\
-\gamma \lambda_{1}-\left(\gamma^{2}+\alpha \beta\right)+\lambda=0 \\
\lambda_{1} \beta-\alpha \gamma=0 \\
\lambda=0
\end{array}\right.
$$

Solve (3.26), we have

Theorem 3.5. $\left(G_{2}, g, J, V\right)$ is not an affine Ricci soliton associated to the connection $\nabla^{1}$.

By (2.64) in [19], we have for $\left(G_{3}, g, J, \nabla^{0}\right)$

$$
\begin{array}{ll}
\widetilde{\rho}^{0}\left(e_{1}, e_{1}\right)=-\gamma a_{3}, & \widetilde{\rho}^{0}\left(e_{1}, e_{2}\right)=0, \\
\widetilde{\rho}^{0}\left(e_{1}, e_{3}\right)=0, & \widetilde{\rho}^{0}\left(e_{2}, e_{2}\right)=-\gamma a_{3}, \\
\widetilde{\rho}^{0}\left(e_{2}, e_{3}\right)=0, & \widetilde{\rho}^{0}\left(e_{3}, e_{3}\right)=0,
\end{array}
$$

where $a_{3}=\frac{1}{2}(\alpha+\beta-\gamma)$. By Lemma 2.24 in [19] and (3.12), we have for $\left(G_{3}, g, J, \nabla^{0}, V\right)$

$$
\begin{array}{ll}
\left(L_{V}^{0} g\right)\left(e_{1}, e_{1}\right)=0, & \left(L_{V}^{0} g\right)\left(e_{1}, e_{2}\right)=0, \\
\left(L_{V}^{0} g\right)\left(e_{1}, e_{3}\right)=-a_{3} \lambda_{2}, & \left(L_{V}^{0} g\right)\left(e_{2}, e_{2}\right)=0, \\
\left(L_{V}^{0} g\right)\left(e_{2}, e_{3}\right)=a_{3} \lambda_{1}, & \left(L_{V}^{0} g\right)\left(e_{3}, e_{3}\right)=0 .
\end{array}
$$

If $\left(G_{3}, g, J, V\right)$ is an affine Ricci soliton associated to the connection $\nabla^{0}$, then by (3.13), we have

$$
\left\{\begin{array}{l}
\gamma a_{3}=0, \\
\lambda_{2} a_{3}=0, \\
\lambda_{1} a_{3}=0, \\
\lambda=0 .
\end{array}\right.
$$

Solve (3.29), we have 
Theorem 3.6. $\left(G_{3}, g, J, V\right)$ is an affine Ricci soliton associated to the connection $\nabla^{0}$ if and only if

(i) $\lambda=0, \alpha+\beta-\gamma=0$,

(ii) $\lambda=0, \alpha+\beta-\gamma \neq 0, \gamma=\lambda_{1}=\lambda_{2}=0$.

By (2.69) in [19], we have for $\left(G_{3}, g, J, \nabla^{1}\right)$

$$
\begin{array}{ll}
\tilde{\rho}^{1}\left(e_{1}, e_{1}\right)=\gamma\left(a_{1}-a_{3}\right), & \widetilde{\rho}^{1}\left(e_{1}, e_{2}\right)=0, \\
\widetilde{\rho}^{1}\left(e_{1}, e_{3}\right)=0, & \widetilde{\rho}^{1}\left(e_{2}, e_{2}\right)=-\gamma\left(a_{2}+a_{3}\right), \\
\widetilde{\rho}^{1}\left(e_{2}, e_{3}\right)=0, & \widetilde{\rho}^{1}\left(e_{3}, e_{3}\right)=0,
\end{array}
$$

where $a_{1}=\frac{1}{2}(\alpha-\beta-\gamma), a_{2}=\frac{1}{2}(\alpha-\beta+\gamma)$. By Lemma 2.27 in [19] and (3.12), we have for $\left(G_{3}, g, J, \nabla^{1}, V\right)$

$$
\begin{aligned}
& \left(L_{V}^{1} g\right)\left(e_{1}, e_{1}\right)=0, \quad\left(L_{V}^{1} g\right)\left(e_{1}, e_{2}\right)=0, \\
& \left(L_{V}^{1} g\right)\left(e_{1}, e_{3}\right)=-\left(a_{2}+a_{3}\right) \lambda_{2}, \quad\left(L_{V}^{1} g\right)\left(e_{2}, e_{2}\right)=0 \text {, } \\
& \left(L_{V}^{1} g\right)\left(e_{2}, e_{3}\right)=\lambda_{1}\left(a_{3}-a_{1}\right), \quad\left(L_{V}^{1} g\right)\left(e_{3}, e_{3}\right)=0 .
\end{aligned}
$$

If $\left(G_{3}, g, J, V\right)$ is an affine Ricci soliton associated to the connection $\nabla^{1}$, then by (3.14), we have

$$
\left\{\begin{array}{l}
\gamma\left(a_{1}-a_{3}\right)+\lambda=0, \\
\left(a_{2}+a_{3}\right) \lambda_{2}=0, \\
-\gamma\left(a_{2}+a_{3}\right)+\lambda=0, \\
\lambda_{1}\left(a_{3}-a_{1}\right)=0, \\
\lambda=0 .
\end{array}\right.
$$

Solve (3.32), we have

Theorem 3.7. $\left(G_{3}, g, J, V\right)$ is an affine Ricci soliton associated to the connection $\nabla^{1}$ if and only if the following statements hold true

(i) $\lambda=0, \gamma \neq 0, \alpha=\beta=0$,

(ii) $\lambda=0, \gamma=0, \alpha \lambda_{2}=0, \lambda_{1} \beta=0$.

By (2.81) in [19], we have for $\left(G_{4}, g, J, \nabla^{0}\right)$

$$
\begin{array}{ll}
\widetilde{\rho}^{0}\left(e_{1}, e_{1}\right)=(2 \eta-\beta) b_{3}-1, & \widetilde{\rho}^{0}\left(e_{1}, e_{2}\right)=0, \\
\widetilde{\rho}^{0}\left(e_{1}, e_{3}\right)=0, & \widetilde{\rho}^{0}\left(e_{2}, e_{2}\right)=(2 \eta-\beta) b_{3}-1, \\
\widetilde{\rho}^{0}\left(e_{2}, e_{3}\right)=\frac{b_{3}-\beta}{2}, & \widetilde{\rho}^{0}\left(e_{3}, e_{3}\right)=0,
\end{array}
$$

where $b_{3}=\frac{\alpha}{2}+\eta$. By Lemma 2.32 in [19] and (3.12), we have for $\left(G_{4}, g, J, \nabla^{0}, V\right)$

$$
\begin{aligned}
& \left(L_{V}^{0} g\right)\left(e_{1}, e_{1}\right)=0, \quad\left(L_{V}^{0} g\right)\left(e_{1}, e_{2}\right)=-\lambda_{2}, \\
& \left(L_{V}^{0} g\right)\left(e_{1}, e_{3}\right)=-b_{3} \lambda_{2}, \quad\left(L_{V}^{0} g\right)\left(e_{2}, e_{2}\right)=2 \lambda_{1}, \\
& \left(L_{V}^{0} g\right)\left(e_{2}, e_{3}\right)=b_{3} \lambda_{1}, \quad\left(L_{V}^{0} g\right)\left(e_{3}, e_{3}\right)=0 .
\end{aligned}
$$

If $\left(G_{4}, g, J, V\right)$ is an affine Ricci soliton associated to the connection $\nabla^{0}$, then by (3.13), we have

$$
\left\{\begin{array}{l}
(2 \eta-\beta) b_{3}-1+\lambda=0 \\
\lambda_{2}=0 \\
\lambda_{1}+(2 \eta-\beta) b_{3}-1+\lambda=0 \\
\lambda_{1} b_{3}+b_{3}-\beta=0 \\
\lambda=0
\end{array}\right.
$$

Solve (3.35), we have

Theorem 3.8. $\left(G_{4}, g, J, V\right)$ is an affine Ricci soliton associated to the connection $\nabla^{0}$ if and only if $\lambda=\lambda_{1}=\lambda_{2}=0, \alpha=0, \beta=\eta$.

By (2.89) in [19], we have for $\left(G_{4}, g, J, \nabla^{1}\right)$

$$
\begin{array}{ll}
\tilde{\rho}^{1}\left(e_{1}, e_{1}\right)=-\left[1+(\beta-2 \eta)\left(b_{3}-b_{1}\right)\right], & \tilde{\rho}^{1}\left(e_{1}, e_{2}\right)=0, \\
\tilde{\rho}^{1}\left(e_{1}, e_{3}\right)=0, & \widetilde{\rho}^{1}\left(e_{2}, e_{2}\right)=-\left[1+(\beta-2 \eta)\left(b_{2}+b_{3}\right)\right], \\
\tilde{\rho}^{1}\left(e_{2}, e_{3}\right)=\frac{\alpha+b_{3}-b_{1}-\beta}{2}, & \widetilde{\rho}^{1}\left(e_{3}, e_{3}\right)=0,
\end{array}
$$


where $b_{1}=\frac{\alpha}{2}+\eta-\beta, b_{2}=\frac{\alpha}{2}-\eta$. By Lemma 2.36 in [19] and (3.12), we have for $\left(G_{4}, g, J, \nabla^{1}, V\right)$

$$
\begin{array}{ll}
\left(L_{V}^{1} g\right)\left(e_{1}, e_{1}\right)=0, & \left(L_{V}^{1} g\right)\left(e_{1}, e_{2}\right)=-\lambda_{2}, \\
\left(L_{V}^{1} g\right)\left(e_{1}, e_{3}\right)=-\left(b_{2}+b_{3}\right) \lambda_{2}-\lambda_{3}, & \left(L_{V}^{1} g\right)\left(e_{2}, e_{2}\right)=2 \lambda_{1}, \\
\left(L_{V}^{1} g\right)\left(e_{2}, e_{3}\right)=\lambda_{1}\left(b_{3}-b_{1}\right), & \left(L_{V}^{1} g\right)\left(e_{3}, e_{3}\right)=0 .
\end{array}
$$

If $\left(G_{4}, g, J, V\right)$ is an affine Ricci soliton associated to the connection $\nabla^{1}$, then by (3.14), we have

$$
\left\{\begin{array}{l}
-\left[1+(\beta-2 \eta)\left(b_{3}-b_{1}\right)\right]+\lambda=0 \\
\lambda_{2}=0 \\
-\left(b_{2}+b_{3}\right) \lambda_{2}-\lambda_{3}=0 \\
\lambda_{1}-\left[1+(\beta-2 \eta)\left(b_{2}+b_{3}\right)\right]+\lambda=0 \\
\lambda_{1}\left(b_{3}-b_{1}\right)+\left(\alpha+b_{3}-b_{1}-\beta\right)=0 \\
\lambda=0
\end{array}\right.
$$

Solve (3.38), we have

Theorem 3.9. $\left(G_{4}, g, J, V\right)$ is not an affine Ricci soliton associated to the connection $\nabla^{1}$.

By (3.5) in [19], we have for $\left(G_{5}, g, J, \nabla^{0}\right), \widetilde{\rho}^{0}\left(e_{i}, e_{j}\right)=0$, for $1 \leq i, j \leq 3$. By Lemma 3.3 in [19] and $(3.12)$, we have for $\left(G_{5}, g, J, \nabla^{0}, V\right)$

$$
\begin{array}{ll}
\left(L_{V}^{0} g\right)\left(e_{1}, e_{1}\right)=0, & \left(L_{V}^{0} g\right)\left(e_{1}, e_{2}\right)=0, \\
\left(L_{V}^{0} g\right)\left(e_{1}, e_{3}\right)=\frac{\beta-\gamma}{2} \lambda_{2}, & \left(L_{V}^{0} g\right)\left(e_{2}, e_{2}\right)=0, \\
\left(L_{V}^{0} g\right)\left(e_{2}, e_{3}\right)=-\frac{\beta-\gamma}{2} \lambda_{1}, & \left(L_{V}^{0} g\right)\left(e_{3}, e_{3}\right)=0 .
\end{array}
$$

If $\left(G_{5}, g, J, V\right)$ is an affine Ricci soliton associated to the connection $\nabla^{0}$, then by (3.13), we have

$$
\left\{\begin{array}{l}
\lambda=0, \\
(\beta-\gamma) \lambda_{2}=0, \\
(\beta-\gamma) \lambda_{1}=0,
\end{array}\right.
$$

Solve (3.40), we have

Theorem 3.10. $\left(G_{5}, g, J, V\right)$ is an affine Ricci soliton associated to the connection $\nabla^{0}$ if and only if one of the following cases occurs

(i) $\lambda=\beta=\gamma=0, \alpha+\delta \neq 0$.

(ii) $\lambda=0, \beta \neq \gamma, \lambda_{1}=\lambda_{2}=0, \alpha+\delta \neq 0, \alpha \gamma+\beta \delta=0$.

By Lemma 3.7 in [19], we have for $\left(G_{5}, g, J, \nabla^{1}\right), \widetilde{\rho}^{1}\left(e_{i}, e_{j}\right)=0$, for $1 \leq i, j \leq 3$. By Lemma 3.6 in [19] and (3.12), we have for $\left(G_{5}, g, J, \nabla^{1}, V\right)$

$$
\begin{array}{ll}
\left(L_{V}^{1} g\right)\left(e_{1}, e_{1}\right)=0, & \left(L_{V}^{1} g\right)\left(e_{1}, e_{2}\right)=0, \\
\left(L_{V}^{1} g\right)\left(e_{1}, e_{3}\right)=-\alpha \lambda_{1}-\gamma \lambda_{2}, & \left(L_{V}^{1} g\right)\left(e_{2}, e_{2}\right)=0, \\
\left(L_{V}^{1} g\right)\left(e_{2}, e_{3}\right)=-\beta \lambda_{1}-\delta \lambda_{2}, & \left(L_{V}^{1} g\right)\left(e_{3}, e_{3}\right)=0 .
\end{array}
$$

If $\left(G_{5}, g, J, V\right)$ is an affine Ricci soliton associated to the connection $\nabla^{1}$, then by (3.14), we have

$$
\left\{\begin{array}{l}
\lambda=0, \\
\alpha \lambda_{1}+\gamma \lambda_{2}=0 \\
\beta \lambda_{1}+\delta \lambda_{2}=0
\end{array}\right.
$$

Solve (3.42), we have

Theorem 3.11. $\left(G_{5}, g, J, V\right)$ is an affine Ricci soliton associated to the connection $\nabla^{1}$ if and only if the following statements hold true

(i) $\lambda=\lambda_{1}=\lambda_{2}=0$,

(ii) $\lambda=0, \lambda_{1} \neq 0, \lambda_{2}=0, \alpha=\beta=0, \delta \neq 0$,

(iii) $\lambda=0, \lambda_{1}=0, \lambda_{2} \neq 0, \delta=\gamma=0, \alpha \neq 0$.

By (3.18) in [19], we have for $\left(G_{6}, g, J, \nabla^{0}\right)$

$$
\widetilde{\rho}^{0}\left(e_{1}, e_{1}\right)=\frac{1}{2} \beta(\beta-\gamma)-\alpha^{2}, \quad \widetilde{\rho}^{0}\left(e_{1}, e_{2}\right)=0,
$$




$$
\begin{array}{ll}
\widetilde{\rho}^{0}\left(e_{1}, e_{3}\right)=0, & \widetilde{\rho}^{0}\left(e_{2}, e_{2}\right)=\frac{1}{2} \beta(\beta-\gamma)-\alpha^{2}, \\
\widetilde{\rho}^{0}\left(e_{2}, e_{3}\right)=\frac{1}{2}\left[-\gamma \alpha+\frac{1}{2} \delta(\beta-\gamma)\right], & \widetilde{\rho}^{0}\left(e_{3}, e_{3}\right)=0 .
\end{array}
$$

By Lemma 3.11 in [19] and (3.12), we have for $\left(G_{6}, g, J, \nabla^{0}, V\right)$

$$
\begin{array}{ll}
\left(L_{V}^{0} g\right)\left(e_{1}, e_{1}\right)=0, & \left(L_{V}^{0} g\right)\left(e_{1}, e_{2}\right)=\alpha \lambda_{2}, \\
\left(L_{V}^{0} g\right)\left(e_{1}, e_{3}\right)=\frac{\gamma-\beta}{2} \lambda_{2}, & \left(L_{V}^{0} g\right)\left(e_{2}, e_{2}\right)=-2 \alpha \lambda_{1}, \\
\left(L_{V}^{0} g\right)\left(e_{2}, e_{3}\right)=\frac{\beta-\gamma}{2} \lambda_{1}, & \left(L_{V}^{0} g\right)\left(e_{3}, e_{3}\right)=0 .
\end{array}
$$

If $\left(G_{6}, g, J, V\right)$ is an affine Ricci soliton associated to the connection $\nabla^{0}$, then by (3.13), we have

$$
\left\{\begin{array}{l}
\frac{1}{2} \beta(\beta-\gamma)-\alpha^{2}+\lambda=0, \\
\alpha \lambda_{2}=0, \\
(\gamma-\beta) \lambda_{2}=0, \\
-\alpha \lambda_{1}+\frac{1}{2} \beta(\beta-\gamma)-\alpha^{2}+\lambda=0, \\
\frac{\beta-\gamma}{2} \lambda_{1}-\gamma \alpha+\frac{1}{2} \delta(\beta-\gamma)=0, \\
\lambda=0 .
\end{array}\right.
$$

Solve (3.45), we have

Theorem 3.12. $\left(G_{6}, g, J, V\right)$ is an affine Ricci soliton associated to the connection $\nabla^{0}$ if and only if

(i) $\lambda=\lambda_{1}=\lambda_{2}=\gamma=\delta=0, \alpha \neq 0, \alpha^{2}=\frac{1}{2} \beta^{2}$,

(ii) $\lambda=\lambda_{1}=\lambda_{2}=\alpha=\beta=\gamma=0, \delta \neq 0$,

(iii) $\lambda=\lambda_{2}=0, \lambda_{1} \neq 0, \alpha=\beta=\gamma=0, \delta \neq 0$,

(iv) $\lambda=\lambda_{2}=0, \lambda_{1} \neq 0, \alpha=\beta=0, \delta \neq 0, \gamma \neq 0, \lambda_{1}=-\delta$,

(v) $\lambda=\alpha=\beta=\gamma=0, \lambda_{2} \neq 0, \delta \neq 0$.

By (3.23) in [19], we have for $\left(G_{6}, g, J, \nabla^{1}\right)$

$$
\begin{array}{ll}
\tilde{\rho}^{1}\left(e_{1}, e_{1}\right)=-\left(\alpha^{2}+\beta \gamma\right), & \widetilde{\rho}^{1}\left(e_{1}, e_{2}\right)=0, \\
\tilde{\rho}^{1}\left(e_{1}, e_{3}\right)=0, & \widetilde{\rho}^{1}\left(e_{2}, e_{2}\right)=-\alpha^{2}, \\
\tilde{\rho}^{1}\left(e_{2}, e_{3}\right)=0, & \widetilde{\rho}^{1}\left(e_{3}, e_{3}\right)=0 .
\end{array}
$$

By Lemma 3.15 in [19] and (3.12), we have for $\left(G_{6}, g, J, \nabla^{1}, V\right)$

$$
\begin{array}{ll}
\left(L_{V}^{1} g\right)\left(e_{1}, e_{1}\right)=0, & \left(L_{V}^{1} g\right)\left(e_{1}, e_{2}\right)=\lambda_{2} \alpha, \\
\left(L_{V}^{1} g\right)\left(e_{1}, e_{3}\right)=-\delta \lambda_{3}, & \left(L_{V}^{1} g\right)\left(e_{2}, e_{2}\right)=-2 \alpha \lambda_{1}, \\
\left(L_{V}^{1} g\right)\left(e_{2}, e_{3}\right)=-\gamma \lambda_{1}, & \left(L_{V}^{1} g\right)\left(e_{3}, e_{3}\right)=0 .
\end{array}
$$

If $\left(G_{6}, g, J, V\right)$ is an affine Ricci soliton associated to the connection $\nabla^{1}$, then by (3.14), we have

Solve (3.48), we have

$$
\left\{\begin{array}{l}
-\left(\alpha^{2}+\beta \gamma\right)+\lambda=0 \\
\lambda_{2} \alpha=0 \\
\delta \lambda_{3}=0 \\
-\alpha \lambda_{1}-\alpha^{2}+\lambda=0 \\
\gamma \lambda_{1}=0 \\
\lambda=0
\end{array}\right.
$$

Theorem 3.13. $\left(G_{6}, g, J, V\right)$ is an affine Ricci soliton associated to the connection $\nabla^{1}$ if and only if the following statements hold true

(i) $\lambda=\alpha=\beta=\lambda_{1}=\lambda_{3}=0, \delta \neq 0$,

(ii) $\lambda=\alpha=\beta=\gamma=\lambda_{3}=0, \delta \neq 0, \lambda_{1} \neq 0$.

By (3.34) in [19], we have for $\left(G_{7}, g, J, \nabla^{0}\right)$

$$
\widetilde{\rho}^{0}\left(e_{1}, e_{1}\right)=-\left(\alpha^{2}+\frac{\beta \gamma}{2}\right), \quad \widetilde{\rho}^{0}\left(e_{1}, e_{2}\right)=0,
$$




$$
\begin{array}{ll}
\widetilde{\rho}^{0}\left(e_{1}, e_{3}\right)=-\frac{1}{2}\left(\gamma \alpha+\frac{\delta \gamma}{2}\right), & \widetilde{\rho}^{0}\left(e_{2}, e_{2}\right)=-\left(\alpha^{2}+\frac{\beta \gamma}{2}\right), \\
\widetilde{\rho}^{0}\left(e_{2}, e_{3}\right)=\frac{1}{2}\left(\alpha^{2}+\frac{\beta \gamma}{2}\right), & \widetilde{\rho}^{0}\left(e_{3}, e_{3}\right)=0 .
\end{array}
$$

By Lemma 3.20 in [19] and (3.12), we have for $\left(G_{7}, g, J, \nabla^{0}, V\right)$

$$
\begin{array}{ll}
\left(L_{V}^{0} g\right)\left(e_{1}, e_{1}\right)=-2 \alpha \lambda_{2}, & \left(L_{V}^{0} g\right)\left(e_{1}, e_{2}\right)=\alpha \lambda_{1}-\beta \lambda_{2}, \\
\left(L_{V}^{0} g\right)\left(e_{1}, e_{3}\right)=\left(\beta-\frac{\gamma}{2}\right) \lambda_{2}, & \left(L_{V}^{0} g\right)\left(e_{2}, e_{2}\right)=2 \beta \lambda_{1}, \\
\left(L_{V}^{0} g\right)\left(e_{2}, e_{3}\right)=\left(\frac{\gamma}{2}-\beta\right) \lambda_{1}, & \left(L_{V}^{0} g\right)\left(e_{3}, e_{3}\right)=0 .
\end{array}
$$

If $\left(G_{7}, g, J, V\right)$ is an affine Ricci soliton associated to the connection $\nabla^{0}$, then by (3.13), we have

$$
\left\{\begin{array}{l}
-\alpha \lambda_{2}-\left(\alpha^{2}+\frac{\beta \gamma}{2}\right)+\lambda=0 \\
\alpha \lambda_{1}-\beta \lambda_{2}=0 \\
\left(\beta-\frac{\gamma}{2}\right) \lambda_{2}-\left(\gamma \alpha+\frac{\delta \gamma}{2}\right)=0 \\
\beta \lambda_{1}-\left(\alpha^{2}+\frac{\beta \gamma}{2}\right)+\lambda=0 \\
\left(\frac{\gamma}{2}-\beta\right) \lambda_{1}+\alpha^{2}+\frac{\beta \gamma}{2}=0 \\
\lambda=0
\end{array}\right.
$$

Solve (3.51), we have

Theorem 3.14. $\left(G_{7}, g, J, V\right)$ is an affine Ricci soliton associated to the connection $\nabla^{0}$ if and only if the following statements hold true

(i) $\lambda=\alpha=\beta=\gamma=0, \delta \neq 0$,

(ii) $\lambda=\alpha=\beta=0, \gamma \neq 0, \lambda_{1}=0, \lambda_{2}=-\delta, \delta \neq 0$,

(iii) $\lambda=\alpha=\gamma=\lambda_{1}=\lambda_{2}=0, \beta \neq 0$.

By (3.42) in [19], we have for $\left(G_{7}, g, J, \nabla^{1}\right)$

$$
\begin{array}{ll}
\tilde{\rho}^{1}\left(e_{1}, e_{1}\right)=-\alpha^{2}, & \tilde{\rho}^{1}\left(e_{1}, e_{2}\right)=\frac{1}{2}(\beta \delta-\alpha \beta), \\
\tilde{\rho}^{1}\left(e_{1}, e_{3}\right)=\beta(\alpha+\delta), & \widetilde{\rho}^{1}\left(e_{2}, e_{2}\right)=-\left(\alpha^{2}+\beta^{2}+\beta \gamma\right), \\
\tilde{\rho}^{1}\left(e_{2}, e_{3}\right)=\frac{1}{2}\left(\beta \gamma+\alpha \delta+2 \delta^{2}\right), & \widetilde{\rho}^{1}\left(e_{3}, e_{3}\right)=0 .
\end{array}
$$

By Lemma 3.24 in [19] and (3.12), we have for $\left(G_{7}, g, J, \nabla^{1}, V\right)$

$$
\begin{array}{ll}
\left(L_{V}^{1} g\right)\left(e_{1}, e_{1}\right)=-2 \alpha \lambda_{2}, & \left(L_{V}^{1} g\right)\left(e_{1}, e_{2}\right)=\alpha \lambda_{1}-\beta \lambda_{2}, \\
\left(L_{V}^{1} g\right)\left(e_{1}, e_{3}\right)=-\alpha \lambda_{1}-\gamma \lambda_{2}-\beta \lambda_{3}, & \left(L_{V}^{1} g\right)\left(e_{2}, e_{2}\right)=2 \beta \lambda_{1}, \\
\left(L_{V}^{1} g\right)\left(e_{2}, e_{3}\right)=-\beta \lambda_{1}-\delta \lambda_{2}-\delta \lambda_{3}, & \left(L_{V}^{1} g\right)\left(e_{3}, e_{3}\right)=0 .
\end{array}
$$

If $\left(G_{7}, g, J, V\right)$ is an affine Ricci soliton associated to the connection $\nabla^{1}$, then by (3.14), we have

$$
\left\{\begin{array}{l}
-\alpha \lambda_{2}-\alpha^{2}+\lambda=0 \\
\alpha \lambda_{1}-\beta \lambda_{2}+\beta \delta-\alpha \beta=0 \\
-\alpha \lambda_{1}-\gamma \lambda_{2}-\beta \lambda_{3}+2 \beta(\alpha+\delta)=0 \\
\beta \lambda_{1}-\left(\alpha^{2}+\beta^{2}+\beta \gamma\right)+\lambda=0 \\
-\beta \lambda_{1}-\delta \lambda_{2}-\delta \lambda_{3}+\beta \gamma+\alpha \delta+2 \delta^{2}=0 \\
\lambda=0
\end{array}\right.
$$

Solve (3.54), we have

Theorem 3.15. $\left(G_{7}, g, J, V\right)$ is an affine Ricci soliton associated to the connection $\nabla^{1}$ if and only if

(i) $\lambda=\alpha=\beta=\gamma=0, \lambda_{2}+\lambda_{3}-2 \delta=0, \delta \neq 0$,

(ii) $\lambda=\alpha=\beta=0, \gamma \neq 0, \lambda_{2}=0, \lambda_{3}=2 \delta, \delta \neq 0$,

(iii) $\lambda=\alpha=0, \delta \neq 0, \beta \neq 0, \lambda_{1}=\beta+\gamma, \lambda_{2}=\delta, \lambda_{3}=\frac{-\gamma \delta+2 \beta \delta}{\beta}, \gamma=\frac{\beta\left(\beta^{2}+\delta^{2}\right)}{\delta^{2}}$. 


\section{AFFINE RICCI SOLITONS ASSOCIATED TO PERTURBED CANONICAL CONNECTIONS AND PERTURBED KOBAYASHI-NOMIZU CONNECTIONS ON THREE-DIMENSIONAL LORENTZIAN LIE GROUPS}

We note that in our classifications in Section 2 always $\lambda=0$. In order to get the affine Ricci soliton with non zero $\lambda$, we introduce perturbed canonical connections and perturbed Kobayashi-Nomizu connections in the following. Let $e_{3}^{*}$ be the dual base of $e_{3}$. We define on $G_{i=1, \ldots, 7}$

$$
\begin{aligned}
& \nabla_{X}^{2} Y=\nabla_{X}^{0} Y+\bar{\lambda} e_{3}^{*}(X) e_{3}^{*}(Y) e_{3}, \\
& \nabla_{X}^{3} Y=\nabla_{X}^{1} Y+\bar{\lambda} e_{3}^{*}(X) e_{3}^{*}(Y) e_{3},
\end{aligned}
$$

where $\bar{\lambda}$ is a non zero real number. Then

$$
\begin{array}{ll}
\nabla_{e_{3}}^{2} e_{3}=\bar{\lambda} e_{3}, & \nabla_{e_{i}}^{2} e_{j}=\nabla_{e_{i}}^{0} e_{j} ; \\
\nabla_{e_{3}}^{3} e_{3}=\bar{\lambda} e_{3}, & \nabla_{e_{i}}^{3} e_{j}=\nabla_{e_{i}}^{1} e_{j} .
\end{array}
$$

where $i$ or $j$ does not equal 3 . We let

$$
\left(L_{V}^{j} g\right)(Y, Z):=g\left(\nabla_{Y}^{j} V, Z\right)+g\left(Y, \nabla_{Z}^{j} V\right)
$$

for $j=2,3$ and vector fields $V, Y, Z$. Then we have for $G_{i=1, \cdots, 7}$

$$
\begin{array}{ll}
\left(L_{V}^{2} g\right)\left(e_{3}, e_{3}\right)=-2 \bar{\lambda} \lambda_{3}, & \left(L_{V}^{2} g\right)\left(e_{j}, e_{k}\right)=\left(L_{V}^{0} g\right)\left(e_{j}, e_{k}\right), \\
\left(L_{V}^{3} g\right)\left(e_{3}, e_{3}\right)=-2 \bar{\lambda} \lambda_{3}, & \left(L_{V}^{3} g\right)\left(e_{j}, e_{k}\right)=\left(L_{V}^{1} g\right)\left(e_{j}, e_{k}\right),
\end{array}
$$

where $j$ or $k$ does not equal 3.

Definition 4.1. $\left(G_{i}, g, J\right)$ is called the affine Ricci soliton associated to the connection $\nabla^{2}$ if it satisfies

$$
\left(L_{V}^{2} g\right)(Y, Z)+2 \widetilde{\rho}^{2}(Y, Z)+2 \lambda g(Y, Z)=0 .
$$

$\left(G_{i}, g, J\right)$ is called the affine Ricci soliton associated to the connection $\nabla^{3}$ if it satisfies

$$
\left(L_{V}^{3} g\right)(Y, Z)+2 \widetilde{\rho}^{3}(Y, Z)+2 \lambda g(Y, Z)=0 .
$$

For $\left(G_{1}, \nabla^{2}\right)$, similar to (3.15), we have

$$
\widetilde{\rho}^{2}\left(e_{2}, e_{3}\right)=\frac{\alpha^{2}+\bar{\lambda} \alpha}{2}, \quad \widetilde{\rho}^{2}\left(e_{j}, e_{k}\right)=\widetilde{\rho}^{0}\left(e_{j}, e_{k}\right),
$$

for the pair $(j, k) \neq(2,3)$. If $\left(G_{1}, g, J, V\right)$ is an affine Ricci soliton associated to the connection $\nabla^{2}$, then by $(4.8)$, we have

$$
\left\{\begin{array}{l}
2 \lambda_{2} \alpha-2 \alpha^{2}-\beta^{2}+2 \lambda=0 \\
\lambda_{1} \alpha=0 \\
-\beta \lambda_{2}+\alpha \beta=0 \\
-2 \alpha^{2}-\beta^{2}+2 \lambda=0 \\
\frac{\beta}{2} \lambda_{1}+\alpha^{2}+\bar{\lambda} \alpha=0 \\
\lambda \lambda_{3}+\lambda=0
\end{array}\right.
$$

Solve (4.11), we have

Theorem 4.2. $\left(G_{1}, g, J, V\right)$ is an affine Ricci soliton associated to the connection $\nabla^{2}$ if and only if $\lambda_{1}=\lambda_{2}=0, \lambda_{3}=-\bar{\lambda}, \alpha=-\bar{\lambda}$, $\beta=0, \lambda=\bar{\lambda}^{2}$.

For $\left(G_{1}, \nabla^{3}\right)$, similar to (3.18), we have

$$
\widetilde{\rho}^{3}\left(e_{2}, e_{3}\right)=\frac{\alpha^{2}+\bar{\lambda} \alpha}{2}, \quad \widetilde{\rho}^{3}\left(e_{j}, e_{k}\right)=\widetilde{\rho}^{1}\left(e_{j}, e_{k}\right),
$$

for the pair $(j, k) \neq(2,3)$. If $\left(G_{1}, g, J, V\right)$ is an affine Ricci soliton associated to the connection $\nabla^{3}$, then by (4.9), we have

$$
\left\{\begin{array}{l}
\lambda_{2} \alpha-\alpha^{2}-\beta^{2}+\lambda=0 \\
-\lambda_{1} \alpha+2 \alpha \beta=0 \\
\lambda_{1} \alpha-\beta \lambda_{2}-\alpha \beta=0 \\
-\alpha^{2}-\beta^{2}+\lambda=0 \\
\beta \lambda_{1}-\alpha \lambda_{2}-\alpha \lambda_{3}+\alpha^{2}+\bar{\lambda} \alpha=0 \\
\bar{\lambda} \lambda_{3}+\lambda=0
\end{array}\right.
$$

Solve (4.13), we have 
Theorem 4.3. $\left(G_{1}, g, J, V\right)$ is not an affine Ricci soliton associated to the connection $\nabla^{3}$.

Proof. By the first and second and fourth equations in (4.13) and $\alpha \neq 0$, we get $\lambda_{2}=0, \lambda_{1}=2 \beta, \lambda=\alpha^{2}+\beta^{2}$, By the third equation in (4.13), we get $\lambda_{1}=\lambda_{2}=\beta=0, \lambda=\alpha^{2}$. By the fifth equation in (4.13), we get $\lambda_{3}=\alpha+\bar{\lambda}$. By the sixth equation in (4.13), we get $\alpha^{2}+\bar{\lambda} \alpha+\bar{\lambda}^{2}=0$. Then $\bar{\lambda}=\alpha=0$, this is a contradiction.

For $\left(G_{2}, \nabla^{2}\right)$, similar to $(3.21)$, we have

$$
\widetilde{\rho}^{2}\left(e_{1}, e_{3}\right)=\frac{-\gamma \bar{\lambda}}{2}, \quad \widetilde{\rho}^{2}\left(e_{j}, e_{k}\right)=\widetilde{\rho}^{0}\left(e_{j}, e_{k}\right)
$$

for the pair $(j, k) \neq(1,3)$. If $\left(G_{2}, g, J, V\right)$ is an affine Ricci soliton associated to the connection $\nabla^{2}$, then by (4.8), we have

$$
\left\{\begin{array}{l}
-\left(\gamma^{2}+\frac{\alpha \beta}{2}\right)+\lambda=0 \\
\lambda_{2} \gamma=0 \\
\alpha \lambda_{2}+2 \gamma \bar{\lambda}=0 \\
-\gamma \lambda_{1}-\left(\gamma^{2}+\frac{\alpha \beta}{2}\right)+\lambda=0 \\
\frac{\alpha}{2} \lambda_{1}+2\left(\frac{\beta \gamma}{2}-\frac{\alpha \gamma}{4}\right)=0 \\
\frac{\lambda}{\lambda} \lambda_{3}+\lambda=0
\end{array}\right.
$$

Solve (4.15), we have

Theorem 4.4. $\left(G_{2}, g, J, V\right)$ is not an affine Ricci soliton associated to the connection $\nabla^{2}$.

For $\left(G_{2}, \nabla^{3}\right)$, similar to $(3.24)$, we have

$$
\widetilde{\rho}^{3}\left(e_{1}, e_{3}\right)=\frac{-\gamma \bar{\lambda}}{2}, \quad \widetilde{\rho}^{3}\left(e_{j}, e_{k}\right)=\tilde{\rho}^{1}\left(e_{j}, e_{k}\right)
$$

for the pair $(j, k) \neq(1,3)$. If $\left(G_{2}, g, J, V\right)$ is an affine Ricci soliton associated to the connection $\nabla^{3}$, then by (4.9), we have

$$
\left\{\begin{array}{l}
-\beta^{2}-\gamma^{2}+\lambda=0, \\
\lambda_{2} \gamma=0, \\
-\alpha \lambda_{2}+\gamma \lambda_{3}-\gamma \bar{\lambda}=0, \\
-\gamma \lambda_{1}-\left(\gamma^{2}+\alpha \beta\right)+\lambda=0 \\
\lambda_{1} \beta-\alpha \gamma=0 \\
\bar{\lambda} \lambda_{3}+\lambda=0
\end{array}\right.
$$

Solve (4.17), we have

Theorem 4.5. $\left(G_{2}, g, J, V\right)$ is not an affine Ricci soliton associated to the connection $\nabla^{3}$.

For $\left(G_{3}, \nabla^{2}\right)$, we have $\widetilde{\rho}^{2}\left(e_{j}, e_{k}\right)=\widetilde{\rho}^{0}\left(e_{j}, e_{k}\right)$, for any pairs $(j, k)$. If $\left(G_{3}, g, J, V\right)$ is an affine Ricci soliton associated to the connection $\nabla^{2}$, then by (4.8), we have

$$
\left\{\begin{array}{l}
-\gamma a_{3}+\lambda=0 \\
\lambda_{2} a_{3}=0 \\
\lambda_{1} a_{3}=0 \\
\bar{\lambda} \lambda_{3}+\lambda=0
\end{array}\right.
$$

Solve (4.18), we have

Theorem 4.6. $\left(G_{3}, g, J, V\right)$ is an affine Ricci soliton associated to the connection $\nabla^{2}$ if and only if the following statements hold true

(i) $a_{3} \neq 0, \lambda_{1}=\lambda_{2}=0, \lambda=\gamma a_{3}, \lambda_{3}=-\frac{\gamma a_{3}}{\bar{\lambda}}$,

(ii) $a_{3}=\lambda=\lambda_{3}=0$. 
For $\left(G_{3}, \nabla^{3}\right)$, we have $\widetilde{\rho}^{3}\left(e_{j}, e_{k}\right)=\widetilde{\rho}^{1}\left(e_{j}, e_{k}\right)$, for any pairs $(j, k)$. If $\left(G_{3}, g, J, V\right)$ is an affine Ricci soliton associated to the connection $\nabla^{3}$, then by (4.9), we have

$$
\left\{\begin{array}{l}
\gamma\left(a_{1}-a_{3}\right)+\lambda=0 \\
\left(a_{2}+a_{3}\right) \lambda_{2}=0, \\
-\gamma\left(a_{2}+a_{3}\right)+\lambda=0 \\
\lambda_{1}\left(a_{3}-a_{1}\right)=0 \\
\bar{\lambda} \lambda_{3}+\lambda=0
\end{array}\right.
$$

Solve (4.19), we have

Theorem 4.7. $\left(G_{3}, g, J, V\right)$ is an affine Ricci soliton associated to the connection $\nabla^{3}$ if and only if one of the following cases occurs

(i) $\gamma=\lambda=\lambda_{3}=0, \alpha \lambda_{2}=0, \beta \lambda_{1}=0$,

(ii) $\gamma \neq 0, \alpha=\beta=\lambda=\lambda_{3}=0$,

(iii) $\gamma \neq 0, \alpha=\beta \neq 0, \lambda_{1}=\lambda_{2}=0, \lambda=\alpha \gamma, \lambda_{3}=-\frac{\alpha \gamma}{\bar{\lambda}}$.

For $\left(G_{4}, \nabla^{2}\right)$, we have

$$
\widetilde{\rho}^{2}\left(e_{1}, e_{3}\right)=\frac{\bar{\lambda}}{2}, \quad \widetilde{\rho}^{2}\left(e_{j}, e_{k}\right)=\widetilde{\rho}^{0}\left(e_{j}, e_{k}\right),
$$

for the pair $(j, k) \neq(1,3)$. If $\left(G_{4}, g, J, V\right)$ is an affine Ricci soliton associated to the connection $\nabla^{2}$, then by $(4.8)$, we have

$$
\left\{\begin{array}{l}
(2 \eta-\beta) b_{3}-1+\lambda=0 \\
\lambda_{2}=0 \\
-b_{3} \lambda_{2}+\bar{\lambda}=0 \\
\lambda_{1}+(2 \eta-\beta) b_{3}-1+\lambda=0 \\
\lambda_{1} b_{3}+b_{3}-\beta=0 \\
\bar{\lambda} \lambda_{3}+\lambda=0
\end{array}\right.
$$

Solve (4.21), we have

Theorem 4.8. $\left(G_{4}, g, J, V\right)$ is not an affine Ricci soliton associated to the connection $\nabla^{2}$.

For $\left(G_{4}, \nabla^{3}\right)$, we have

$$
\widetilde{\rho}^{3}\left(e_{1}, e_{3}\right)=\frac{\bar{\lambda}}{2}, \quad \widetilde{\rho}^{3}\left(e_{j}, e_{k}\right)=\widetilde{\rho}^{1}\left(e_{j}, e_{k}\right),
$$

for the pair $(j, k) \neq(1,3)$. If $\left(G_{4}, g, J, V\right)$ is an affine Ricci soliton associated to the connection $\nabla^{3}$, then by (4.9), we have

$$
\left\{\begin{array}{l}
-\left[1+(\beta-2 \eta)\left(b_{3}-b_{1}\right)\right]+\lambda=0, \\
\lambda_{2}=0, \\
-\left(b_{2}+b_{3}\right) \lambda_{2}-\lambda_{3}+\bar{\lambda}=0, \\
\lambda_{1}-\left[1+(\beta-2 \eta)\left(b_{2}+b_{3}\right)\right]+\lambda=0, \\
\lambda_{1}\left(b_{3}-b_{1}\right)+\left(\alpha+b_{3}-b_{1}-\beta\right)=0, \\
\bar{\lambda} \lambda_{3}+\lambda=0 .
\end{array}\right.
$$

Solve (4.23), we have

Theorem 4.9. $\left(G_{4}, g, J, V\right)$ is not an affine Ricci soliton associated to the connection $\nabla^{3}$.

For $\left(G_{5}, g, J, \nabla^{2}\right), \widetilde{\rho}^{2}\left(e_{i}, e_{j}\right)=0$, for $1 \leq i, j \leq 3$. If $\left(G_{5}, g, J, V\right)$ is an affine Ricci soliton associated to the connection $\nabla^{2}$, then by $(4.8)$, we have

$$
\left\{\begin{array}{l}
\lambda=0 \\
(\beta-\gamma) \lambda_{2}=0 \\
(\beta-\gamma) \lambda_{1}=0 \\
\bar{\lambda} \lambda_{3}+\lambda=0
\end{array}\right.
$$

Solve (4.24), we have

Theorem 4.10. $\left(G_{5}, g, J, V\right)$ is an affine Ricci soliton associated to the connection $\nabla^{2}$ if and only if the following statements hold true

(i) $\gamma \neq \beta, \lambda=\lambda_{1}=\lambda_{2}=\lambda_{3}=0, \alpha+\delta \neq 0, \alpha \gamma+\beta \delta=0$,

(ii) $\lambda=\beta=\gamma=0, \alpha+\delta \neq 0, \lambda_{3}=0$. 
For $\left(G_{5}, g, J, \nabla^{3}\right), \widetilde{\rho}^{3}\left(e_{i}, e_{j}\right)=0$, for $1 \leq i, j \leq 3$. If $\left(G_{5}, g, J, V\right)$ is an affine Ricci soliton associated to the connection $\nabla^{3}$, then by (4.9), we have

$$
\left\{\begin{array}{l}
\lambda=0, \\
\alpha \lambda_{1}+\gamma \lambda_{2}=0, \\
\beta \lambda_{1}+\delta \lambda_{2}=0, \\
\bar{\lambda} \lambda_{3}+\lambda=0 .
\end{array}\right.
$$

Solve (4.25), we have

Theorem 4.11. $\left(G_{5}, g, J, V\right)$ is an affine Ricci soliton associated to the connection $\nabla^{3}$ if and only if

(i) $\lambda=\lambda_{1}=\lambda_{2}=\lambda_{3}=0$,

(ii) $\lambda=\lambda_{2}=\lambda_{3}=\alpha=\beta=0, \lambda_{1} \neq 0, \delta \neq 0$,

(iii) $\lambda=0, \lambda_{1}=\lambda_{3}=0, \lambda_{2} \neq 0, \delta=\gamma=0, \alpha \neq 0$.

For $\left(G_{6}, \nabla^{2}\right)$, we have

$$
\widetilde{\rho}^{2}\left(e_{1}, e_{3}\right)=\frac{\delta \bar{\lambda}}{2}, \quad \widetilde{\rho}^{2}\left(e_{j}, e_{k}\right)=\widetilde{\rho}^{0}\left(e_{j}, e_{k}\right),
$$

for the pair $(j, k) \neq(1,3)$. If $\left(G_{6}, g, J, V\right)$ is an affine Ricci soliton associated to the connection $\nabla^{2}$, then by (4.8), we have

$$
\left\{\begin{array}{l}
\frac{1}{2} \beta(\beta-\gamma)-\alpha^{2}+\lambda=0 \\
\alpha \lambda_{2}=0 \\
(\gamma-\beta) \lambda_{2}+2 \delta \bar{\lambda}=0 \\
-\alpha \lambda_{1}+\frac{1}{2} \beta(\beta-\gamma)-\alpha^{2}+\lambda=0 \\
\frac{\beta-\gamma}{2} \lambda_{1}-\gamma \alpha+\frac{1}{2} \delta(\beta-\gamma)=0 \\
\bar{\lambda} \lambda_{3}+\lambda=0
\end{array}\right.
$$

Solve (4.27), we have

Theorem 4.12. $\left(G_{6}, g, J, V\right)$ is an affine Ricci soliton associated to the connection $\nabla^{2}$ if and only if the following statements hold true

(i) $\alpha=\beta=0, \delta \neq 0, \gamma \neq 0, \lambda=\lambda_{3}=0, \lambda_{1}=-\delta, \lambda_{2}=-\frac{2 \delta \bar{\lambda}}{\gamma}$,

(ii) $\alpha \neq 0, \lambda_{1}=\lambda_{2}=\gamma=\delta=0, \lambda=\alpha^{2}-\frac{1}{2} \beta^{2}, \lambda_{3}=-\frac{\lambda}{\lambda}$.

For $\left(G_{6}, \nabla^{3}\right)$, we have

$$
\tilde{\rho}^{3}\left(e_{1}, e_{3}\right)=\frac{\delta \bar{\lambda}}{2}, \quad \widetilde{\rho}^{3}\left(e_{j}, e_{k}\right)=\tilde{\rho}^{1}\left(e_{j}, e_{k}\right),
$$

for the pair $(j, k) \neq(1,3)$. If $\left(G_{6}, g, J, V\right)$ is an affine Ricci soliton associated to the connection $\nabla^{3}$, then by (4.9), we have

$$
\left\{\begin{array}{l}
-\left(\alpha^{2}+\beta \gamma\right)+\lambda=0 \\
\lambda_{2} \alpha=0 \\
-\delta \lambda_{3}+\delta \bar{\lambda}=0 \\
-\alpha \lambda_{1}-\alpha^{2}+\lambda=0 \\
\gamma \lambda_{1}=0 \\
\bar{\lambda} \lambda_{3}+\lambda=0
\end{array}\right.
$$

Solve (4.29), we have

Theorem 4.13. $\left(G_{6}, g, J, V\right)$ is an affine Ricci soliton associated to the connection $\nabla^{3}$ if and only if $\alpha \neq 0, \lambda_{1}=\lambda_{2}=\gamma=\delta=0, \lambda=\alpha^{2}$, $\lambda_{3}=-\frac{\alpha^{2}}{\bar{\lambda}}$.

For $\left(G_{7}, \nabla^{2}\right)$, we have

$$
\widetilde{\rho}^{2}\left(e_{1}, e_{3}\right)=\frac{1}{2}\left(\beta \bar{\lambda}-\alpha \gamma-\frac{\delta \gamma}{2}\right), \quad \widetilde{\rho}^{2}\left(e_{2}, e_{3}\right)=\frac{1}{2}\left(\delta \bar{\lambda}+\alpha^{2}+\frac{\beta \gamma}{2}\right), \quad \widetilde{\rho}^{2}\left(e_{j}, e_{k}\right)=\widetilde{\rho}^{0}\left(e_{j}, e_{k}\right),
$$


for the pair $(j, k) \neq(1,3),(2,3)$. If $\left(G_{7}, g, J, V\right)$ is an affine Ricci soliton associated to the connection $\nabla^{2}$, then by (4.8), we have

$$
\left\{\begin{array}{l}
-\alpha \lambda_{2}-\left(\alpha^{2}+\frac{\beta \gamma}{2}\right)+\lambda=0 \\
\alpha \lambda_{1}-\beta \lambda_{2}=0 \\
\left(\beta-\frac{\gamma}{2}\right) \lambda_{2}+\beta \bar{\lambda}-\left(\gamma \alpha+\frac{\delta \gamma}{2}\right)=0 \\
\beta \lambda_{1}-\left(\alpha^{2}+\frac{\beta \gamma}{2}\right)+\lambda=0 \\
\left(\frac{\gamma}{2}-\beta\right) \lambda_{1}+\delta \bar{\lambda}+\alpha^{2}+\frac{\beta \gamma}{2}=0 \\
\bar{\lambda} \lambda_{3}+\lambda=0
\end{array}\right.
$$

Solve (4.31), we have

Theorem 4.14. $\left(G_{7}, g, J, V\right)$ is an affine Ricci soliton associated to the connection $\nabla^{2}$ if and only if one of the following cases occurs

(i) $\alpha=\beta=0, \gamma \neq 0, \delta \neq 0, \lambda=0, \lambda_{1}=-\frac{2 \delta \bar{\lambda}}{\gamma}, \lambda_{2}=-\delta, \lambda_{3}=0$,

(ii) $\alpha \neq 0, \lambda_{1}=\lambda_{2}=\beta=\gamma=0, \lambda=\alpha^{2}, \delta \neq 0, \bar{\lambda}=-\frac{\alpha^{2}}{\delta}, \lambda_{3}=\delta$.

For $\left(G_{7}, \nabla^{3}\right)$, we have

$$
\tilde{\rho}^{3}\left(e_{1}, e_{3}\right)=\alpha \beta+\beta \delta+\frac{\beta \bar{\lambda}}{2}, \quad \widetilde{\rho}^{3}\left(e_{2}, e_{3}\right)=\frac{1}{2}\left(\beta \gamma+\alpha \delta+2 \delta^{2}+\delta \bar{\lambda}\right), \quad \tilde{\rho}^{3}\left(e_{j}, e_{k}\right)=\widetilde{\rho}^{1}\left(e_{j}, e_{k}\right),
$$

for the pair $(j, k) \neq(1,3),(2,3)$. If $\left(G_{7}, g, J, V\right)$ is an affine Ricci soliton associated to the connection $\nabla^{3}$, then by (4.9), we have

$$
\left\{\begin{array}{l}
-\alpha \lambda_{2}-\alpha^{2}+\lambda=0, \\
\alpha \lambda_{1}-\beta \lambda_{2}+\beta \delta-\alpha \beta=0, \\
-\alpha \lambda_{1}-\gamma \lambda_{2}-\beta \lambda_{3}+2 \beta(\alpha+\delta+\bar{\lambda})=0, \\
\beta \lambda_{1}-\left(\alpha^{2}+\beta^{2}+\beta \gamma\right)+\lambda=0, \\
-\beta \lambda_{1}-\delta \lambda_{2}-\delta \lambda_{3}+\beta \gamma+\alpha \delta+2 \delta^{2}+\delta \bar{\lambda}=0, \\
\bar{\lambda} \lambda_{3}+\lambda=0 .
\end{array}\right.
$$

Solve (4.33), we have

Theorem 4.15. $\left(G_{7}, g, J, V\right)$ is an affine Ricci soliton associated to the connection $\nabla^{3}$ if and only if the following statements hold true

(i) $\lambda=\alpha=\beta=\gamma=\lambda_{3}=0, \delta \neq 0, \lambda_{2}=2 \delta+\bar{\lambda}$,

(ii) $\alpha=\beta=\lambda=\lambda_{2}=\lambda_{3}=0, \gamma \neq 0, \delta \neq 0, \bar{\lambda}=-2 \delta$,

(iii) $\alpha=\lambda=\lambda_{3}=0, \beta \neq 0, \delta \neq 0, \lambda_{1}=\beta+\gamma, \lambda_{2}=\delta, \bar{\lambda}=\frac{\gamma \delta-2 \beta \delta}{\beta}, \gamma=\frac{\beta^{3}+\beta \delta^{2}}{\delta^{2}}$,

(iv) $\alpha \neq 0, \beta=\gamma=\delta=\lambda_{1}=\lambda_{2}=0, \lambda=\alpha^{2}, \lambda_{3}=-\frac{\alpha^{2}}{\bar{\lambda}}$,

(v) $\alpha \neq 0, \beta=\gamma=\lambda_{1}=\lambda_{2}=0, \lambda=\alpha^{2}, \delta \neq 0, \lambda_{3}=\alpha+2 \delta+\bar{\lambda}, \bar{\lambda}^{2}+(\alpha+2 \delta) \bar{\lambda}+\alpha^{2}=0$.

Proof. We know that $\alpha \gamma=0$ and $\alpha+\delta \neq 0$.

Case i) $\quad \alpha=0$, then $\delta \neq 0$. By (4.33), we have $\lambda=\lambda_{3}=0$ and

$$
\left\{\begin{array}{l}
\beta\left(\lambda_{2}-\delta\right)=0 \\
-\gamma \lambda_{2}+2 \beta \delta+\beta \bar{\lambda}=0 \\
\beta \lambda_{1}-\left(\beta^{2}+\beta \gamma\right)=0 \\
-\beta \lambda_{1}-\delta \lambda_{2}+\beta \gamma+2 \delta^{2}+\delta \bar{\lambda}=0
\end{array}\right.
$$

Case i)-a) $\quad \beta=0$, then by (4.34), we have $\gamma \lambda_{2}=0$ and $\lambda_{2}=2 \delta+\bar{\lambda}$.

Case i)-a)-1) $\gamma=0$, we get (i).

Case i)-a)-2) $\gamma \neq 0$, we get $\lambda_{2}=0$ and $\bar{\lambda}=-2 \delta$. So we have (ii).

Case i)-b) $\quad \beta \neq 0$, then by (4.34), we have $\lambda_{1}=\beta+\gamma, \lambda_{2}=\delta, \bar{\lambda}=\frac{\gamma \delta-2 \beta \delta}{\beta}$. By the fourth equation in (4.34), we get $\gamma=\frac{\beta^{3}+\beta \delta^{2}}{\delta^{2}}$ and this is (iii).

Case ii) $\quad \alpha \neq 0$, so $\gamma=0$.

Case ii)-a) $\quad \beta=0$, by (4.33), we get $\lambda_{1}=\lambda_{2}=0, \lambda=\alpha^{2}, \delta \lambda_{3}=\alpha \delta+2 \delta^{2}+\delta \bar{\lambda}, \lambda_{3}=-\frac{\alpha^{2}}{\bar{\lambda}}$.

Case ii)-a)-1) $\delta=0$, we get (iv).

Case ii)-a)-2) $\delta \neq 0$, we get (v). 
Case ii)-b) $\quad \beta \neq 0$, we get $\alpha \lambda_{2}+\beta \lambda_{1}-\beta^{2}=0$ and $\alpha \lambda_{1}-\beta \lambda_{2}+\beta(\delta-\alpha)=0$. So get

$$
\left\{\begin{array}{l}
\lambda_{1}=\beta-\frac{\alpha \beta \delta}{\alpha^{2}+\beta^{2}}, \\
\lambda_{2}=\frac{\beta^{2} \delta}{\alpha^{2}+\beta^{2}} \\
\lambda=\frac{\alpha \beta^{2} \delta}{\alpha^{2}+\beta^{2}}+\alpha^{2} \\
\lambda_{3}=\bar{\lambda}+\alpha+2 \delta+\frac{\alpha^{2} \delta}{\alpha^{2}+\beta^{2}} .
\end{array}\right.
$$

Using (4.35) and the fifth equation in (4.33), we get $\beta^{2}\left(\alpha^{2}-\alpha \delta+\delta^{2}+\beta^{2}\right)+\alpha^{2} \delta^{2}=0$, so we get $\beta=0$ and this is a contradiction. So we have no solutions in this case.

\section{CONFLICTS OF INTEREST}

The author declares no conflicts of interest.

\section{FUNDING}

This research was funded by National Natural Science Foundation of China: No. 11771070.

\section{ACKNOWLEDGMENTS}

The author was supported in part by NSFC No. 11771070. The author is deeply grateful to the referees for their valuable comments and helpful suggestions.

\section{REFERENCES}

[1] Z.M. Balogh, J.T. Tyson, E. Vecchi, Intrinsic curvature of curves and surfaces and a Gauss-Bonnet theorem in the Heisenberg group, Math. Z. 287 (2017), 1-38.

[2] W. Batat, K. Onda, Algebraic Ricci solitons of three-dimensional Lorentzian Lie groups, J. Geom. Phys. 114 (2017), 138-152.

[3] G. Calvaruso, Three-dimensional homogeneous generalized Ricci solitons, Mediterr. J. Math. 14 (2017), 216.

[4] G. Calvaruso, Homogeneous structures on three-dimensional Lorentzian manifolds, J. Geom. Phys. 57 (2007), $1279-1291$.

[5] L.A. Cordero, P.E. Parker, Left-invariant Lorentzian metrics on 3-dimensional Lie groups, Rend. Mat. Appl. 17 (1997), $129-155$.

[6] M. Crasmareanu, A new approach to gradient Ricci solitons and generalizations, Filomat 32 (2018), 3337-3346.

[7] F. Etayo, R. Santamaría, Distinguished connections on ( $J^{2}= \pm 1$ )-metric manifolds, Arch. Math. (Brno) 52 (2016), 159-203.

[8] N. Halammanavar, K. Devasandra, Kenmotsu manifolds admitting Schouten-van Kampen connection, Facta Univ. Ser. Math. Inform. 34 (2019), $23-34$.

[9] R.S. Hamilton, The Ricci flow on surfaces, in: J.A. Isenberg, (Ed.), Mathematics and general relativity, Contemp. Math., 71, Amer. Math. Soc., Providence, RI, Santa Cruz, CA, 1988, pp. 237-262.

[10] Y. Han, A. De, P. Zhao, On a semi-quasi-Einstein manifold, J. Geom. Phys. 155 (2020), 103739.

[11] S. Hui, R. Prasad, D. Chakraborty, Ricci solitons on Kenmotsu manifolds with respect to quarter symmetric non-metric $\phi$-connection, Ganita 67 (2017), $195-204$.

[12] S.Y. Perktas, A. Yildiz, On quasi-Sasakian 3-manifolds with respect to the Schouten-van Kampen connection, Int. Elec. J. Geom. 13 (2020), $62-74$.

[13] Q. Qu, Y. Wang, Multiply warped products with a quarter-symmetric connection, J. Math. Anal. Appl. 431 (2015), $955-987$.

[14] A.N. Siddiqui, B.Y. Chen, O. Bahadir, Statistical solitons and inequalities for statistical warped product submanifolds, Mathematics 7 (2019), 797.

[15] S. Sular, C. Özgür, Warped products with a semi-symmetric metric connection, Taiwanese J. Math. 15 (2011), 1701-1719.

[16] S. Sular, C. Özgür, Warped products with a semi-symmetric non-metric connection, Arab. J. Sci. Eng. 36 (2011), $461-473$.

[17] Y. Wang, Multiply warped products with a semisymmetric metric connection, Abstr. Appl. Anal. 2014 (2014), 742371.

[18] Y. Wang, Curvature of multiply warped products with an affine connection, Bull. Korean Math. Soc. 50 (2013), $1567-1586$.

[19] Y. Wang, Canonical connections and algebraic Ricci solitons of three-dimensional Lorentzian Lie groups, arxiv:2001.11656.2020.

[20] Y. Wang, S. Wei, Gauss-Bonnet theorems in the affine group and the group of rigid motions of the Minkowski plane, Sci. China Math. 2020.

[21] T. Wu, S. Wei, Y. Wang, Gauss-Bonnet theorems and the Lorentzian Heisenberg group, 2020, Available from: https://www.researchgate.net/publication/ 345360459_GAUSS-BONNET_THEOREMS_AND_THE_LORENTZIAN_HEISENBERG_GROUP. 